\title{
Design and Implementation of Engineering Investigation Information System based on GIS
}

\author{
Qiong Yin ${ }^{1}$ \\ ${ }^{1}$ Kunming Metallurgy College, Kunming, Yunnan, 610051
}

\section{KEYWORDS: GIS; Engineering Investigation Information System; System Design}

\begin{abstract}
The rapid development of urbanization, urban engineering survey has a huge impact, traditional urban reconnaissance production management tools has exposed its limitations, geographic information systems involved, unprecedented space for development of urban engineering survey. How to effectively and scientifically manage huge engineering survey data, how to quickly achieve high-quality engineering survey data collection, timely new history, retrieval and query, how to quickly implement engineering survey data graphs, tables, etc. output, these are city engineering survey the core issue of most concern. In this paper, many of the above problems, for the urban engineering survey of the characteristics of the survey and analysis of the data, the completion of the survey data classification and coding, standardized survey data, the establishment of urban engineering survey database, on this basis, the completion of the city engineering survey and design of information systems and realized.
\end{abstract}

\section{Introduction}

GIS is a use of computer hardware, software and network technology, urban spatial data and non-spatial data collection, management, processing, analysis, update and provide applications and to handle a variety of urban spatial entities and their relationship main technical systems. Geographic Information System as a branch of the geographic information system, which in addition to the general characteristics of geographic information systems, but also has its own uniqueness. Mainly reflected in the following aspects:

It includes the basic geographic factors and a variety of data sources, environmental, social and economic, in time for a long time phase, the structure is multilayered, nature and mound presence, "spatial orientation" and "property" of the points, both graphically oriented vector data, but also as a source of remote sensing raster data; in terms of clients, it is necessary to consider the municipal executives, the professional sector and the needs of the public inquiry, but also to meet the management, analysis and planning of the price prediction different user needs, therefore, to the city information system service object of the multilayer high demands.

Based on the characteristics of the city, in a sense, Geographic Information Systems Geographic Information Systems in the human scale geoscience application, and therefore its accuracy is correspondingly high. At the same time, accelerate the process of urbanization and urban development and updated information requirements also will improve the timeliness side to ensure good current situation of the information.

Geographic information system is necessary to achieve the modernization of management, but also to achieve the development and optimization strategy, it is bound to have a complete set of analysis, evaluation, forecasting and optimization models, and with management, analysis and 
evaluation of a variety of functions forecast, etc., than general geographic information system should be more integrated and more advanced intelligence needs.

\section{Data Analysis of Engineering Investigation Information System}

The primary task of information systems engineering investigation is to establish a city survey database, the original geological data, hydrological data, environmental data, project information, etc. for unified management. Establishment of a database, you must first determine the standardization of data, there is no standard, it is difficult to unify disparate data, data conversion and can not be reused in different formats, different possible structures more unified planning database, field codes, expression Wait.

Urban data on investigation process involved a wide range of complex structure, and for the huge amounts of data, but the nature of their mathematical point of view, the same general geological data, can be summarized as "qualitative data" (in the database with "character type "means) and" quantitative data "(in the database with" numerical "representation).

The main survey process maps are the following:

1) Geological plan, reflecting the level of outcrop geological phenomena and distribution;

2) Drilling histogram, reflecting a variety of geological phenomena and vertical distribution comparison;

3) Engineering geological profile, reflecting the vertical distribution of geological phenomena;

In the geographical letter from the system, the transmission characteristics of data authenticity and uniqueness of influence, such as distribution network, were maps, but the engineering survey information system, most of the data has variability, by the experience and human factors such as relatively many. Source data is multiple sources, such as: measurement and calculation data from the field of drilling, geophysical exploration, test data and test chamber and the like. How they are organically organize effectively store, manage and retrieve directly affect the efficiency of the database application and the whole engineering survey information system. Thus, geographic classification and coding of information is a very important basic work.

For ease of operation, improve the entry, query, retrieval efficiency and enhanced data sharing, data must type the name of the code, that is a set of ordered character string instead of a noun Jun, terminology and even a special phrase. Code is the common language of the display unit is classified information, proofread, statistics and key retrieval.

Main function of the code identification, classification and sorting tri-sec, which is the most basic function of the differential function within the system, a code can uniquely identify an entity, on the contrary, an entity can only have a standardized code, that is a matter code, a code Kazuyoshi allowed weight, garbled, wrong code, therefore, to be in line with national standards and code words.

\section{Engineering Investigation Information System}

Characteristics and target systems engineering survey, the system performs the following functions:

Engineering survey and GIS database. Number of arrested library as a system-wide basis, and all other operations are based on the database. Therefore, the design and construction of the database is essential.

Attribute data entry and management. In the geographical letter from the system, the attribute data as digital spatial information exists, the attribute data entry and effective management, including data entry, query, backup, search, import and export permission settings. 
Tube graphical raw data and graphical results of data. This part of the focus on solving the graphics data, including raw data and results of data, such as topographic maps, geological maps, engineering geology floor plan, project profiles, drilling histogram management, and seamless operation attribute data and graphic data.

Engineering survey results generated maps. Including drilling buckle-shaped map, cross-sectional view of engineering geology, engineering geology floorplan and a variety of in-situ test results of FIG other cities' process design and construction, urban planning and construction of the most commonly used basic maps.

Engineering survey results table generation. Including the most basic and generate a variety of in-situ testing, indoor tables and statistical analysis of the results of the test tables and other information systems engineering survey results it wants most of the table, as well as pre-project (summary) operator to generate the results of the table. Auxiliary analysis system Include contour, three-dimensional geological maps and expert systems.

Design engineering geological database system, in essence, is to reflect the long geological information about the survey area, data, organized into conforming to a particular database management system (such as Microsoft Access) data required by the model data structure. In order to make the design out of the database can satisfy the needs of engineering geological survey, before the specific logical structure of the database during the design, various geological model must be established in the system analysis are converted to the corresponding series of numbers caught mode. These data mode is called mode data survey area, they are merely a true reflection of the objective situation of the survey area, without considering the organization of data and the efficiency of database access specific database management systems. Then, to convert these reflect the objective situation of the survey area data pattern into the data format required by Microsoft Access that logical data model. Geological survey information the complexity and diversity of its decisions can not be a unified data schema model. The transformation zone survey data analyzed by the model is a geologic model, integrated process. It's roughly the steps are: first of all the properties in a variety of geological model entity set, according to the source and value way into many categories, and then compare the various categories, which combined with the kind or properties of the same name, and then related to "standard", "standard" and the current production records for comparison table, grouped into several categories, to give series of data patterns. Each data pattern is a new entity set, and each data item within the new entity set corresponds to a property, a collection of these data patterns can be used as engineering geological database global data model to look at, on the one hand it has become this database the standard format of the data collection; the other hand, form the basis of the database file system design.

Urban Engineering Investigation Information System is used to manage various types of urban engineering survey data for the survey industry's production and management services, and can provide a variety of integrated engineering geological data for the planning, design, provide decision-makers and decision-making based on monitoring data. Using the popular, user-friendly interface and meet production engineering investigation habits, develop a convenient, fast entry module.

It provides bidirectional conversion between DGN and DWG; provide $\mathrm{N}$ bidirectional conversion and standard text format, providing two-way conversion and other major Chinese DG G $\mathrm{S}$ work platform file format. 


\section{Integrated Query}

Provides various forms of query method, it is to provide a structured (SQL) queries, and provide the results of the query output.

Screen chart system (CAD) based on Micro-station platform to achieve a variety of geological prospecting process $\mathrm{Bu}$ handmade chart function. Offers a variety of engineering geology symbol library, linear library, seed files, and a variety of templates, establish a sound and friendly charting interface,

Automatically generate a variety of graphs, tables and database platforms use Micro-station in situ, interior and other data to automatically generate all kinds of engineering diagrams.

The use of engineering investigations in situ testing, indoor testing, mapping and other spatial data, combined with experience and standard data on the characteristics of urban engineering geological environmental suitability and stability as possible discrimination. Provide valid parameters for the survey and design and construction, engineering, building design and construction. Such as seismic liquefaction, pile foundation design parameter calculation, slope stability parameter meter (estimate) operator and so on.

\section{Conclusion}

This article focuses on the city information engineering survey, design and construction of the system database, the database is the foundation of the whole system, and therefore the data collection, updating, storage, and building a database and other rigorous design process, ensuring high data quality requirements, achieve a seal engineering survey spatial data and attribute data integration management, while the security of the database strict and effective design, ensure the safety and lawful use of data. The system can meet the survey industry, production management, and from the survey data entry, powder processing, retrieving and updating of various types of survey results to charts generated function, and is able to carry out various forms of survey data access query; at the same time this system also has its more powerful secondary analysis functions for urban planning, design and construction to provide information on the basis of information and decision-making, therefore, the system has strong practical value.

\section{Reference}

[1] David Frost J, Jean Lou A Chameau. Proceedings of Geographic Information Systems and Their Application in Geotechnical Earthquake Engineering [C]. Atlanta: American Society of Civil Engineers, 2003: 17-21.

[2] Emmi Jose Macari, James R Martin, Thomas L.Brandon (ASCEA.M.). Liquefaction Potential of Puertorican]. David Frost J, Jean-Lou A Château. Proceedings of Geographic Information Systems and Their Application in Geotechnical Earthquake Engineering [C]. Atlanta: American Society of Civil Engineers, 1993.72-77.

[3] James R Martin. Development of geographical information system (GIS) for seismic hazard study of Charleston, SC [A], David Frost J, Jean-Lou A Chameau. Proceedings of Geographic Information Systems and Their Application in Geotechnical Earthquake Engineering [C]. Atlanta: American Society of Civil Engineers, 1993.77-82.

[4] Kiersch, G A-Development of engineering geology in western United States [J]. Engineering Geology, Jan 2001. 